\title{
Einladung zur Falldiskussion am Donnerstag, 28.10.2021
}

\section{6:00-17:30 Uhr via ZOOM}

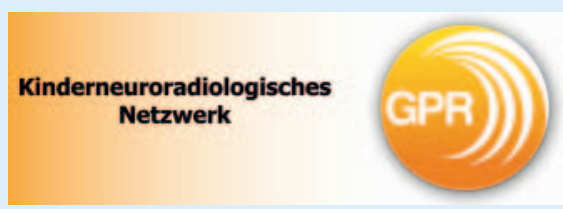

Liebe Kolleginnen und Kollegen,

die pädiatrische Neuroradiologie bildet einen wichtigen und komplexen Teil der pädiatrischen Radiologie und der Neuroradiologie. Erfahrungsgemäß gibt es in jedem Zentrum meist 1-2 (Neuro-/pädiatrische) Radiologen/Radiologinnen, die sich schwerpunktmäßig mit diesem Gebiet befassen, sodass der kollegiale Austausch begrenzt ist und in kleineren Zentren möglicherweise auch gar nicht möglich ist.

Da jeden von uns jedoch immer wieder interessante und herausfordernde Fälle erreichen, ist ein kollegialer Austausch nicht nur zur Falldiskussion im Rahmen der Diagnosefindung von Interesse, sondern auch zur Fort- und Weiterbildung anzustreben.

\section{Wir freuen uns daher, Sie zur interaktiven Falldiskussion auf ZOOM begrüßen zu dürfen.}

Das Programm finden Sie auf der Homepage der GPR (kinder-radiologie.org).
Die Teilnahme ist kostenfrei, eine Anmeldung auf der GPR-Homepage jedoch erforderlich.

Nach der Registrierung erhalten Sie eine Bestätigungs-E-Mail mit Informationen über die Teilnahme am Meeting.

Für Rückfragen steht Ihnen die Geschäftsstelle unter der Mailadresse buero@kinderradiologie.org gerne zur Verfügung.

Wir freuen uns über Ihre Teilnahme!

Mit herzlichen Grüßen

Dr. med. Gabriele Hahn, Universitätskinderradiologie Dresden 